\title{
29
}

\section{Congenital Breast Malformations}

\author{
Armand Lucas and Serdar Nasir
}

\section{Summary}

Congenital breast deformities pose a challenging dilemma in plastic surgery. The expression of the abnormality may be complete, and anatomic components are often missing, deformed, or weakened. Most breasts are naturally asymmetrical. These asymmetries may include discrepancies in breast size and shape, location of the nipple-areola complex, the inframammary fold, or skeletal abnormalities (see Figure 29.1). Breast abnormalities can cause emotional and psychological trauma, resulting in social maladjustment and associated behavioral problems. The majority of patients are young healthy individuals who seek aesthetic restoration of their deformities.

Preoperative evaluation begins with a thorough medical and surgical history, particularly related to breast disease. Physical examination includes identification of chest wall or musculoskeletal deformities; shape, symmetry, and volume of both breasts; the presence and degree of ptosis; the position of the inframammary folds; and anomalies of the nipple-areola complex. Most of these anomalies require a multistage surgical approach, and the patient must be extensively counseled.

\section{Abbreviations}

DIEP Deep inferior epigastric perforator flap

LDF Latissimus dorsi muscle myocutaneous flap

SGAP Superior gluteal artery perforator flap

TRAM Transverse rectus abdominis myocutaneous flap

\section{Poland's Syndrome}

Poland's syndrome was first described by Alfred Poland as a medical student in London in 1841. Although Lallemand and Froriep presented patients with similar anomalies, Alfred Poland's description is by far the most precise and comprehensive. ${ }^{7}$ The sine qua non of Poland's syndrome is the absence of a sternocostal portion of the pectoralis major muscle, a hypoplastic or absent breast and/or nipple-areola complex, and may include upper-extremity abnormalities such as hypoplasia of the hand, forearm, and arm. This may also include complete or incomplete syndactyly and short fingers. The chest wall can have abnormalities with depressed ribs and occasional absence of the latissimus dorsi muscle, serratus anterior muscle, and external oblique muscle. Absence of the pectoralis major muscle occurs with an incidence of approximately 1:7,000 to $1: 1,00,000$ live births. It commonly affects males 
in a 2:1 to 3:1 ratio and has a right-sided predisposition in $60-75 \%$ of cases. ${ }^{8}$ The etiology of this condition is unknown, and there is no genetic pattern or familial predisposition. The most accepted theory refers to a circulatory defect of the subclavian artery around the 45th gestational day. ${ }^{9}$ This has been termed the "subclavian artery supply disruption sequence" and is supported by Merlob et al.'s evaluation of subclavian artery diameter and flow. ${ }^{18}$

Poland's syndrome has a large spectrum of clinical presentations which correlate well with the degree of functional impairment and cosmetic deformity. The surgical correction of Poland's syndrome deformity requires addressing four separate issues: (1) Addressing the chest wall deformity, (2) addressing the breast deformity in females, (3) surgical correction of any upperextremity deformities, and, lastly, (4) nipple-areola reconstruction.

The ideal reconstruction goals in female Poland's anomaly patients require achieving breast symmetry, correcting the chest wall deformity, recreating the anterior axillary fold, and providing an adequate infraclavicular fullness for aesthetic and psychosocial aspects. ${ }^{3}$ In female Poland's syndrome, some clinical situations correcting the breast contour may be enough to camouflage the deformity without the need for extensive muscle flap transposition (i.e.,latissimus dorsi flap) (Figure 29.1).

The latissimus dorsi muscle flap and muscul cutaneous components have been used for patients with Poland's syndrome since first introduced for breast reconstruction. Latissimus dorsi transposition reestablishes the anterior axillary fold, filling in the supraclavicular hollows, particularly in males with thin subcutaneous tissue. This replaces the pectoralis major muscle, which is strongly associated with the male gender. In females, it provides excellent coverage for a breast implant or a tissue expander. In patients in their developing adolescent years, an expander implants such as the Becker implant can be used temporarily and later on exchanged for a permanent implant when the patient has completed growth. The timing of this reconstruction, at least with a muscle transposition, should be seriously considered at age 5-6 during the preschool years before serious anxieties and psychological problems develop. To create the anterior axillary fold with a latissimus dorsi flap, some surgeons advocate dividing the latissimus insertion tendon on the humerus and transferring it to a more anterior position to increase its projection across the axilla. ${ }^{16}$ In females performing a latissimus dorsi muscle transfer without an expander or a mammary prosthesis may result in an inadequate volume in most cases. If the patient has a larger tissue defect or is older and has significant lower abdominal tissue, a TRAM flap, even a deep inferior epigastric flap (DIEP) flap, or a superficial inferior epigastric artery flap can be transferred as an option. Despite the aesthetically pleasing results that can be achieved with the latissimus dorsi transposition, substantial donor-site morbidity can exist because of the large incision needed to harvest and transfer the
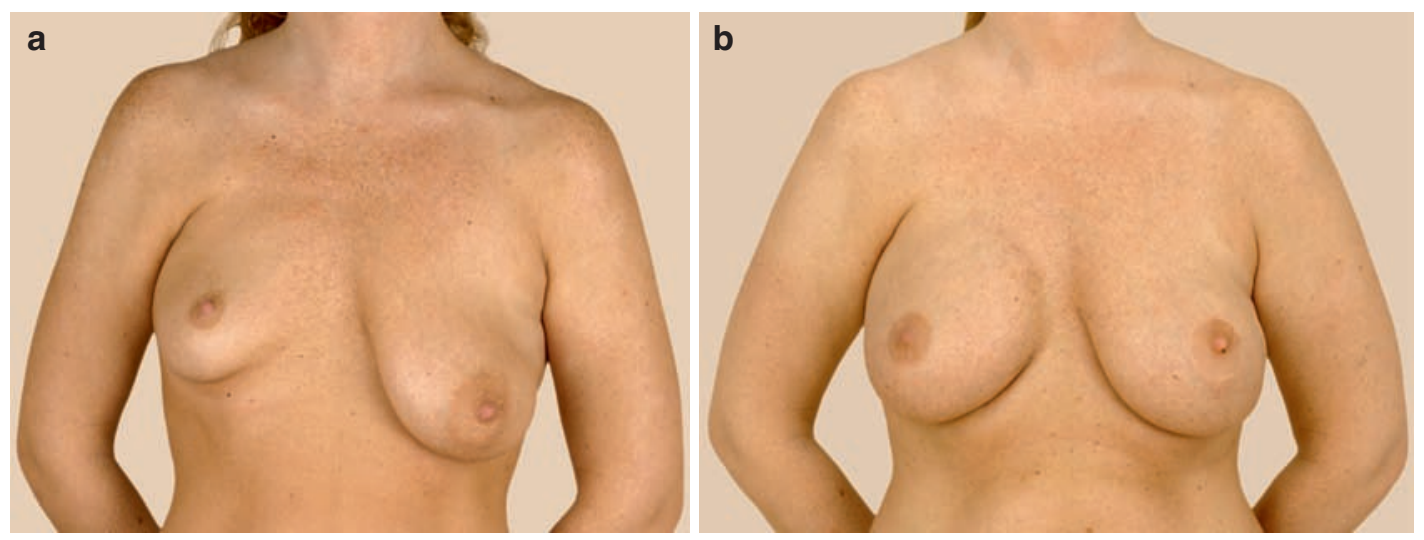

Figure 29.1. (a) A 32 year-old female with Poland's syndrome. Right breast corrected with a 350-cc gel implant and a simultaneous left breast mastopexy. No need for latissimus transposition. (b) Postoperative result 3 years later. 
flaps. ${ }^{17}$ Borschel et al described a two-stage minimally invasive technique that requires only a single small incision in the axilla for the muscle dissection and implant placement. In the first stage of this technique, a tissue expander is placed endoscopically to increase the size of the skin envelope. After a period of expansion, at a second stage, the tissue expander is removed endoscopically and a permanent saline implant is placed. The latissimus dorsi muscle can then be transferred. For some patients, the opportunity to reduce the dermasite scar through endoscopic techniques is a more attractive option. In situations where the syndrome includes hypoplasia of the latissimus dorsi muscle, free tissue transfer procedures can provide excellent results. The free superior gluteal myocutaneous flap and free rectus flaps have been reported by various authors. ${ }^{14,24}$ However, the use of the TRAM flap particularly if tripedicle may have detrimental effects on the abdominal wall of young patients. Donor-site morbidity can include abdominal hernias, bulging, prolonged pain, and anesthesia of the involved tissues. For those reasons, perforator free flaps have provide an excellent alternative method of autologous reconstruction for this syndrome. The DIEP ${ }^{13}$ and the superior gluteal artery flap (SGAP), ${ }^{9}$ are currently used for breast reconstruction by Poland's syndrome. The advantage of the DIEP flap is that it lessens the amount of fascial dissection and rectus muscle dissection at the donor site. Thus, it may decrease postoperative pain and lessen recovery, and some studies have shown a slightly lower incidence of hernia formation and bulging compared to the TRAM flap. ${ }^{10}$ The Superior gluteal artery prefactor flap (SGAP) may be used if the patient does not have adequate abdominal tissue, as in the case of many younger patients. However, there is a high chance for buttock contour donor-site deformity requiring secondary revisions. Furthermore, perforator flaps need advanced microsurgical skill, and this procedures takes longer operative time.

A separate deformity from Poland's syndrome was described by Spear et al. in 2004, and it was coined the anterior thoracic hypoplasia syndrome. This deformity and Poland's syndrome share similar traits; however, in the anterior thoracic hypoplasia syndrome, the pectoralis major muscle is completely normal unlike the situation in Poland's syndrome where the pectoralis hypoplasia or missing sternocostal head is the defining feature. Poland's syndrome has varying degrees of severity and involvement, and it may or may not have involvement of the upper extremity, but the anterior thoracic hypoplasia syndrome tissue deficiencies are similar with regard to chest and breast size and can occur on the contralateral side. The etiology is unknown. This second syndrome is more easily corrected by the use of breast augmentation to increase breast size and projection of both breasts. Augmentation mammoplasty is the preferred method, because it is simpler compared with alternative flap procedures.

\section{Axillary Breast}

The human breast develops from ectoderm during the fifth week of gestation. The earliest identifiable breast tissue is the mammary ridge, which expands bilaterally from the axilla in a caudal direction to the groin. Breast tissue can develop anywhere along this embryologic breast line. One of the most common sites of extra mammary breast tissue is the axilla but it can also be found in unusual locations such as the scapula, thigh, posterior leg, or the labia majora (Figure 29.2). Ectopic breast tissue is at risk for similar benign and malignant problems as normal breasts, which include fibrocystic disease, mastitis, fibroadenomas, atypical hyperplasia, and carcinoma. ${ }^{6}$ Marshall in 1994 reported an increased incidence of cancer in aberrant breast tissue. Accessory axillary breast tissues are common embryologic alterations found in about $1-6 \%$ of women, and they often manifest bilaterally. ${ }^{1}$ Axillary breast tissue presenting as a palpable axillary mass can undergo monthly menstrual changes as well as tenderness, swelling, difficulty with shoulder motion and irritation from clothing. All symptoms may be aggravated, becoming more apparent during puberty and pregnancy. Ectopic cancers located in the axilla seem to present with more extensive disease at an earlier age, suggesting that aberrant tissue may be at increased risk for malignant change. The treatment of axillary breast tissue is generally elliptical excision, although axillary breast tissue has also been reportedly removed with ultrasound-assisted liposuction to avoid a large scar. ${ }^{5}$ 

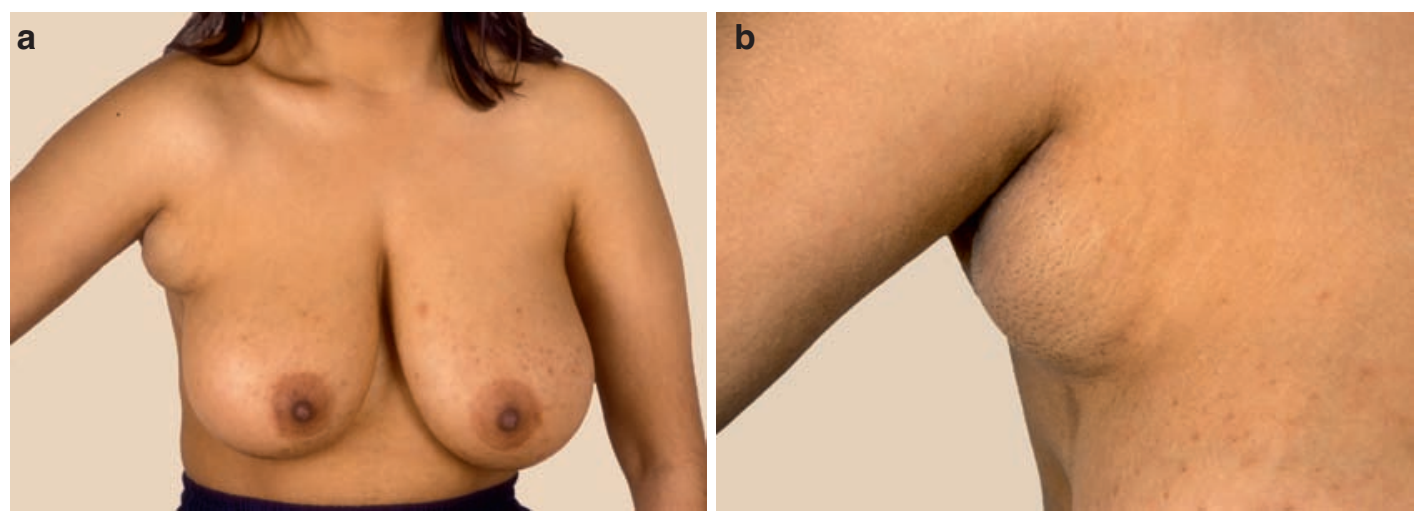

Figure 29.2. (a) Axillary breast; (b) detail.

\section{Polythelia}

Supranumerary nipples or polythelia (Figure 29.3) can be found along the line from the axilla to the groin. This anomaly is the most common anomaly in congenital breast deformities present in about $5 \%$ of the general population and can be found in both men and women. ${ }^{22} \mathrm{~A}$ remnant of milk lines after improper regression of the mammary ridges at approximately 3 months of gestation causes accessory nipples. ${ }^{2}$ The most common site for an abnormal nipple is inferior to the normal breast but superior to the lower abdominal inguinal regions. In approximately $50 \%$ of the patients, it can be bilateral. It has also been described outside the milk line, such as the scapula, posterior thigh, head, and neck. ${ }^{25}$ Although polythelia rarely causes more than an aesthetic concern to the patient, sporadic polythelia may be associated with other congenital deformities including kidney urologic abnormalities. This anomaly has also been associated with testicular cancer. It has been reported that male patients with supernumerary nipples have an increased risk of testicular tumors, but the actual relative risk remains uncertain because of the relatively lower incidence of both diseases. ${ }^{11}$ These pigmented lesions in the milk line should be excised before puberty, particularly in young girls, because recession may require larger tissue excision if there is glandular growth. There have been a few reported cases of cancerous degeneration of this accessory nipple-areola complex tissue, and that provides an additional justification for excision of these lesions. ${ }^{22}$

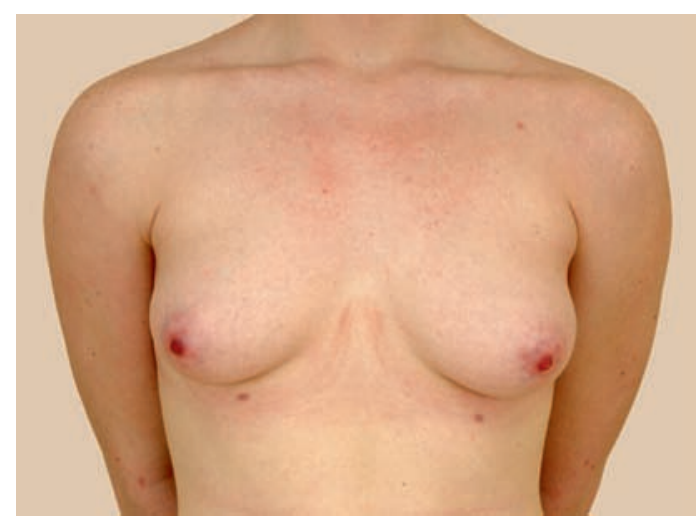

Figure 29.3. Polythelia.

\section{Tuberous Breast}

Tuberous breast syndrome is a rare anomaly presenting in adolescent mammary development. It was initially described by Rees and Aston, ${ }^{20}$ and it is characterized by a constricted base, hypoplastic breast tissue, a herniated nippleareola complex, an elevated inframammary fold, and a deficient skin envelope in the lower pole vertically and transversally. Its etiology is unknown. There is no genetic or familial incidence, and a proposed cause of this tuberous deformity is the presence of a ring of fascia that constricts radial growth. It is hypothesized that this constricting ring of superficial fascia limits growth of the lower pole and results in a high inframammary fold. Mammary tissue herniates through a hiatus in the fascia located directly 

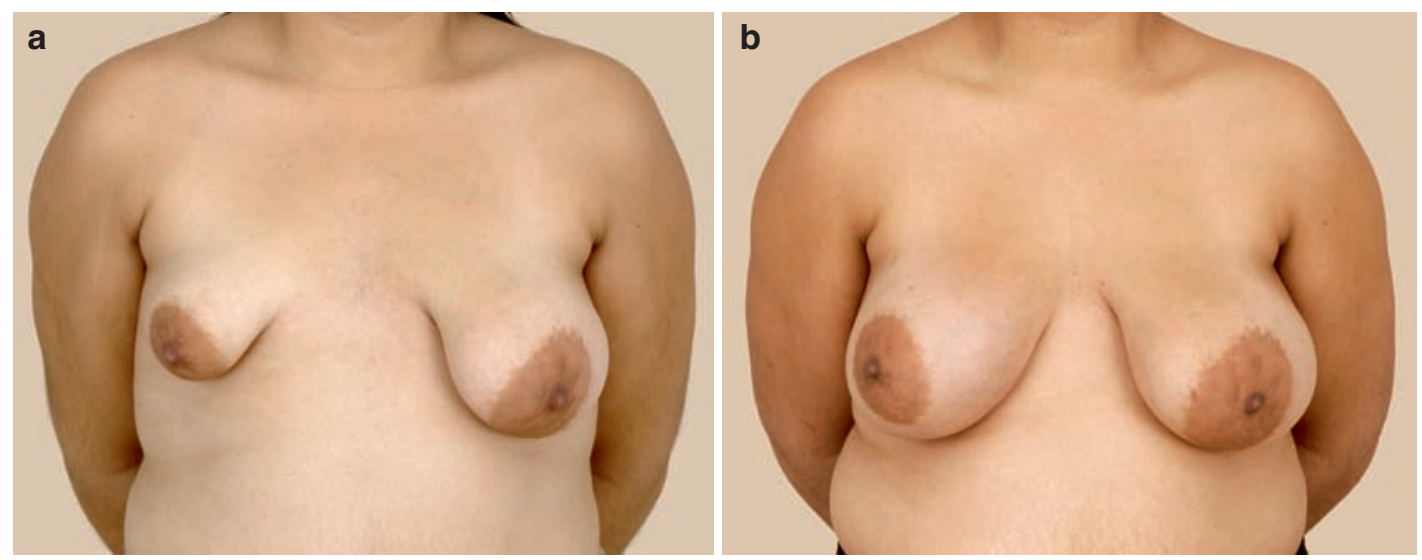

Figure 29.4. (a) A 27-year-old female with right tuberous breast deformity, $5 \mathrm{ft} 11$ in., $220 \mathrm{lb}$. (b). Deformity corrected with 3 months of tissue expansion and later replacement with 600-cc gel prosthesis on right and 500-cc augmentation on left. Postoperative result at 1 year.

deep to the nipple-areolar complex. ${ }^{26}$ This breast tissue deficiency results in the tuberous or "potatolike" appearance of the breast that may involve only the lower pole or may involve the breast circumferentially. This deformity becomes evident during adolescence and can generate significant psychological disturbances. Generally, volume deficiency of tuberous breast is at least $27 \%$ less than the contralateral breast, and in many cases, it is bilateral to a variable degree. Grolleau et al. ${ }^{12}$ reported that in $28 \%$ of tuberous breast deformities, the breast was reported to be hypertrophic.

The classifications reported in the literature are based primarily on the shape of the deformity. The von Heimburg classification is divided into four categories based on the degree of hypoplasia of the base of the breast. In this classification, type 1 and type 3 breasts may be considered the same, because the amount of skin envelope is simply dependent on the size of the glandular tissue itself. Grolleau et al. ${ }^{12}$ reduced the classification group to three classes based on the degree of hypoplasia at the base of the breast. Type 1 is characterized by hypoplasia of the lower medial quadrant; type 2 breasts are hypoplastic on both lower quadrants, and the areola points downward; and type 3 has hypoplasia in all four quadrants. ${ }^{12}$

There are multiple surgical techniques described for correction of tuberous breast deformity. The main goals are to restore the base dimensions, correct hypoplasia of the lower quadrants, reposition the inframammary fold, correct the nipple- areola complex herniation by reducing the size of the areola, correct ptosis, and provide as close a symmetry as possible with the contralateral breast (Figure 29.4).

The timing of reconstructive surgery for most congenital breast deformities is important to the final outcome. Surgery at a young age can damage the breast bud and cause major growth deformities. In most situations, corrective surgery should not be undertaken until the breast is fully developed by the age of 15 or 16 years.

The tuberous breast with herniation of breast tissue through a constricted fascial ring may be approached through a periareolar incision, allowing for areolar reduction and release of the constricting rim. The inframammary approach is another effective way to release this band and adjust the inferior position of the breast. The chosen technique depends on careful study of the deformities of the breast to achieve proper correction.

The superior pedicle mammoplasty with lateral dermal glandular flap techniques also provides filling of the lower quadrant with breast tissue. The Maillard Z-plasty technique may be used for type 1 and type 2 deformities, and in this technique, the inframammary fold is lowered to a normal position, and the inferior portion of the breast is reshaped by overlapping the elevated inferior skin flaps in the fashion of a Z-plasty. ${ }^{15}$ The Ribeiro technique may be used for type 2 and type 3 deformities. In this technique, an inferior flap is made by dividing the lower pole of the breast from the upper pole and the nipple-areolar complex. This flap is then folded over itself to 
give the inferior pole projection and as close to normal in appearance as possible. ${ }^{21}$ Choupina et al. ${ }^{4}$ reported the successful use of this technique in combination with silicone implant placement for type three deformities. In the Puckett technique, the inferior flap is based just deep to the nipple-areola complex; it is elevated, and a submuscular implant is placed, and the breast is unfurled anteriorly over the implant, transferring tissue bulk to the inferior pole. ${ }^{19}$

Tissue expansion has become, in many ways, the mainstay of the treatment of tuberous breast deformities (Figure 29.4a). The constricted base of the breast is approached through the inframammary incision; multiple cruciate incisions are made along the base of the breast to destroy and eliminate the constricted bands, and a tissue expander is placed in the submammary position. The patient then undergoes a period of expansion, which may range from 2 to 4 months depending on the size needed to match the contralateral breast, and then at a second stage, has the tissue expander removed, the pocket capsule adjusted, and a silicone implant placed to match the contralateral side. In many situations, a contralateral augmentation or mastopexy is performed to improve symmetry (see Figure 29.4b).

\section{Congenital Absence of the Breast}

Isolated absence of the breast was first described by Froriep in 1839. Unilateral absence can be seen together with Poland's syndrome, bilateral absence is rare, and most cases of amastia are a component of other developmental syndromes such as UllrichTurner syndrome or the AREDYLD syndrome. ${ }^{23}$ In these situations, breast reconstruction techniques undertaken are similar to those used in breast cancer surgery. That is, the use of the transverse rectus abdominus myocutaneous flap or latissimus dorsi flap plus an implant. Tissue expansion with subsequent placement of a silicone implant is also a viable option.

\section{References}

1. Bhatnagar KP, Ramsaroop L, Bhatnagar KP, Satyapal KS, Singh B.Dorsal scapular breast in a woman. Plast Reconstr Surg. 2003;112:571-574.
2. Bland KI, Copeland EM. The Breast: Comprehensive Management of Benign and Malignant Disorders. 3rd ed. Philadelphia, PA: Elsevier; 2004.

3. Borschel GH, Izenberg PH, Cederna PS. Endoscopically assisted reconstruction of male and female Poland syndrome. Plast Reconstr Surg. 2002;109:1536-1543.

4. Choupina M, Malheiro E, Pinho C, et al. Tuberous breast: a surgical challenge. Aesthet Plast Surg. 2002;26:50-53.

5. Emsen IM. Treatment with ultrasound-assisted liposuction of accessory axillary breast tissues. Aesthet Plast Surg. 2006;30:251-252.

6. Evans DM, Guyton DP. Carcinoma of the axillary breast. J Surg Oncol. 1995;59:190-195.

7. Fokin AA, Robicsek F. Poland's syndrome revisited. Ann Thorac Surg. 2002;74:2218-2225.

8. Freire-Maia N, Chautard EA, Opitz JM, Freire-Maia A, Quelce-Salgado A. The Poland syndrome-clinical and genealogical data, dermatoglyphic analysis, and incidence. Hum Hered. 1973;23:97-104.

9. Gautam AK, Allen RJ Jr, LoTempio MM, et al. Congenital breast deformity reconstruction using perforator flaps. Ann Plast Surg. 2007;58:353-358.

10. Gill PS, Hunt JP, Guerra AB, et al. A 10-year retrospective review of 758 DIEP flaps for breast reconstruction. Plast Reconstr Surg. 2004;113:1153-1160.

11. Goedert JJ, McKeen EA, Javadpour N, Ozols RF, Pottern LM, Fraumeni JF Jr. Polythelia and testicular cancer. Ann Intern Med. 1984;101:646-647.

12. Grolleau JL, Lanfrey E, Lavigne B, Chavoin JP, Costagliola $M$. Breast base anomalies: treatment strategy for tuberous breasts, minor deformities, and asymmetry. Plast Reconstr Surg. 1999;104:2040-2048.

13. Liao HT, Cheng MH, Ulusal BG, Wei FC. Deep inferior epigastric perforator flap for successful simultaneous breast and chest wall reconstruction in a Poland anomaly patient. Ann Plast Surg. 2005;55:422-426.

14. Longaker MT, Glat PM, Colen LB, Siebert JW. Reconstruction of breast asymmetry in Poland's hest-wall deformity using microvascular free flaps. Plast Reconstr Surg. 1997;99:429-436.

15. Maillard GF. A Z-mammaplasty with minimal scarring. Plast Reconstr Surg. 1986;77:66-76.

16. Marks MW, Argenta LC, Izenberg PH, Mes LG. Management of the chest-wall deformity in male patients with Poland's syndrome. Plast Reconstr Surg. 1991;87:674-678; discussion 679-681.

17. May JW Jr, Lukash FN, Gallico GG III. Latissimus dorsi harvest for free and pedicled tissue transfer. In: Bostwick J, Felmont FE, Nahai F, Barrows S, eds. Endoscopic Plastic Surgery. St Louis, MO: Quality Medical Publishing; 1995.

18. Parker DL, Mitchell PR, Holmes GL. Poland-Mobius syndrome. J Med Genet. 1981;18:317-320.

19. Puckett CL, Concannon MJ. Augmenting the narrowbased breast: the unfurling technique to prevent the double-bubble deformity. Aesthet Plast Surg. 1990;14:15-19.

20. Rees TD, Aston SJ. The tuberous breast. Clin Plast Surg. 1976;3:339-347.

21. Ribeiro L, Canzi W, Buss A Jr, Accorsi A Jr. Tuberous breast: a new approach. Plast Reconstr Surg. 1998;101:42-50; discussion 51-42.

22. Schmidt H. Supernumerary nipples: prevalence, size, sex and side predilection - a prospective clinical study. Eur J Pediatr. 1998;157:821-823. 
23. Trier WC. Complete breast absence. Case report and review of the literature. Plast Reconstr Surg. 1965;36:431-439.

24. Tvrdek M, Kletensky J, Svoboda S. Aplasia of the breastreconstruction using a free tram flap. Acta Chir Plast. 2001;43:39-41.
25. Velanovich V.Ectopic breast tissue, supernumerary breasts, and supernumerary nipples. S Med J. 1995;88:903-906.

26. von Heimburg D, Exner K, Kruft S, Lemperle G. The tuberous breast deformity: classification and treatment. Br J Plast Surg. 1996;49:339-345. 